\title{
Evaluation of a Cooperative ARQ Protocol for Delay-Tolerant Vehicular Networks ${ }^{1}$
}

\author{
Julian Morillo-Pozo $^{1}$, Oscar Trullols ${ }^{1}$, Jose M. Barceló ${ }^{1}$, Jorge Garcia-Vidal ${ }^{1}$, \\ ${ }^{1}$ Computer Architecture Department (DAC), \\ Techinal University of Catalonia (UPC), \\ Campus Nord, c/Jordi Girona, 1-3, \\ 08034 Barcelona, Spain \\ \{imorillo, trullols, joseb, jorge\}@ac.upc.edu
}

\begin{abstract}
This paper evaluates a Cooperative ARQ protocol to be used in delay-tolerant vehicular networks. The scenario consists in cars downloading information from Access Points along a road. The key difference between proposed Cooperative ARQ protocols is when the cooperation takes place. Simply C-ARQ cooperation occurs in a packet-by-packet basis. In this proposal, that we call DC-ARQ (Delayed Cooperative ARQ), the cooperation is delayed until cars are out of the coverage area of the Access Point. The scheme has been evaluated through simulations. A comparison of DC-ARQ with a baseline case in which no cooperation is used has been performed under different vehicle densities scenarios.
\end{abstract}

Keywords: Cooperative systems, Disruptive Tolerant Networks, Vehicular Adhoc Networks.

\section{Introduction}

Vehicular Ad-hoc NETworks (VANETs) are a particular case of MANETs in which nodes are vehicles that move following specific patterns (i.e. roads). Important applications of VANETs are: Transportation-related applications and Convenience and Personalized applications including Internet access, hot-spots access, gaming, sharing files or P2P services.

In this paper we focus on delay-tolerant applications, in which cars download information from Access Points (AP) placed on the road.

VANETs are networks characterized by intermittent connectivity and rapid changes in their topology. In the considered scenario, vehicles accessing an AP have few seconds to download information in an environment with high losses. Measurements of UDP and TCP transmissions of vehicles in a highway passing in front of an

\footnotetext{
${ }^{1}$ This work has been supported by Spanish Ministry of Science and Technology under grant TSI2007-66869-C02-01 and by the NoE EuroFGI of the VI FP of the UE under VNET-3 project.
} 
AP moving at different speeds report losses on the order of $50-60 \%$ depending on the nominal sending rate and vehicle speed; see [1].

In this harsh environment, innovative communication techniques are needed. We believe that cooperative techniques can be beneficial in order to improve the performance of this type of networks and applications. The main objectives of this paper are to test this hypothesis and to compare two possible solutions on this direction.

The main contribution of this work is an evaluation of the Delayed Cooperative ARQ (C-ARQ) scheme to be used in vehicular networks where cars download delaytolerant information from AP on the road, suffering an intermittent connectivity. In a first general scheme, not addressed to VANET, cooperation among nodes is established in a packet-by-packet basis, [10]. DC-ARQ, on the contrary, cooperation is established in the dark areas, where connectivity with the AP is lost. In this way, AP contact times

To evaluate the scheme we have simulated using NS [2] the case of a straight road with sparse AP giving intermittent connectivity to vehicles. We have also compared the scheme with a baseline case in which no cooperation is used.

The main outcome of our research is that the DC-ARQ protocol can effectively reduce the packet losses of transmissions from access points to cars in a platoon increasing the delivery ratio of the network. This improvement is more spectacular when the vehicle density is high, but also for lower densities DC-ARQ has shown to be an effective technique.

\section{Related work}

A performance study in term of losses when vehicles enter the coverage of an access point in a highway and exchange UDP and TCP packets is presented in [1].

Most of the work related to opportunistic vehicular networks deal with opportunistic forwarding strategies, in which nodes schedule the forwarding of packets according to opportunities; see [3], [4], [5]. This scheduling may be based on: historical path likelihoods, [3], packet replication [4], or on the expected packet forwarding delay, [5]. These proposals take as a point of reference epidemic routing [6]. Their main objective is to optimize contact opportunities to forward packets in intermittent scenarios, but they do not consider how to optimize the transference of such information given that you have contacted another node.

Cooperative ARQs are schemes which increase link reliability in data link protocols through the use of node cooperation; see [7], [8], [9], [10]. In [8] authors describe a scheme for improving loss resilience with diversity, focusing on wireless local area networks (WLANs). In [7], authors propose a two-phase communication using a relay node. In [9] authors present a generalization of Hybrid-ARQ where retransmitted packets do not need to come from the original source radio but could instead be sent by relays that overhear the transmission. The job reported in [10] presents a novel frame exchange mechanism between a node and its cooperators for C-ARQ/FC (Cooperative ARQ with Frame Combining).

DC-ARQ, previously proposed in [11], follows the Infostation model, in which nodes transport data and deliver their information during contact times; see [12]. In 
the case of DC-ARQ hot-spots distributed along roads act as Infostations, while gaps between Infostations are used to interchange packets with other nodes.

Packet-by-packet Cooperative ARQ has as objective to recover immediately lost packets from neighbor vehicles at the cost of bandwidth at contact times. This scheme has sense if different channels are used: one to download information from AP and other to cooperate. Delayed Cooperative ARQ (DC-ARQ) has as objective of not wasting bandwidth at contact times and waits to dark areas in which vehicles have not connectivity with AP to cooperate and recover as much packets as possible from neighbor nodes. This scheme has been proposed and tested in a simple prototype with 3 vehicles in an urban scenario, [11]. The tests were performed using ping packets sent from an AP to each of the 3 vehicles and then, these vehicles recovered losses after leaving AP coverage. The work showed that vehicles were able to recover in some experiments around $50 \%$ of the packets lost. However, we did not test the prototype in denser scenarios due to the difficulty in scaling experiments. Furthermore, cooperation scenarios were not compared not cooperation scenarios.

\section{Cooperative ARQ for Delay-Tolerant Vehicular Networks}

Consider Figure 1 in which vehicles want to download information from the Internet through AP distributed along a road. Due to the harsh conditions produced in VANET, the losses produced in such environment are high. Reference [1] reports experiments on a highway in which vehicles passing in front of an AP moving at different speeds have losses on the order of $50-60 \%$ depending on the nominal sending rate and vehicle speed.

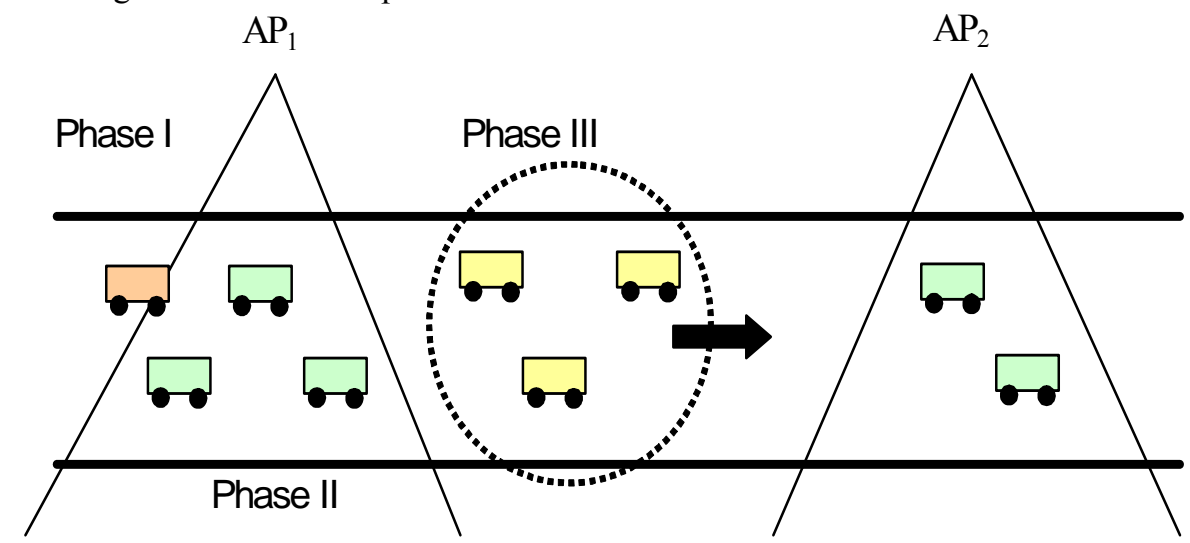

Figure 1. VANET scenario.

AP acts as custodians in a Delay/Disruptive Tolerant Network (DTN), [13], keeping files for nodes. It is not the purpose of this paper to define the DTN architecture. We will assume that there is a server that schedules which files are stored at each AP and which and how packets are sent to the vehicles.

Although and end-to-end ARQ mechanism is needed to recover packets, we may use the broadcast nature of the media to improve packet recovery. Cooperative 
techniques may improve performance in terms of packet losses. When a vehicle receives a packet, its neighbor nodes may independently receive the packet. In this way, some of the vehicles in the platoon traveling with the destination node may have some of the lost packets. Nodes, then, may cooperate to recover packet losses minimizing the number of end-to-end retransmissions.

\subsection{Delayed Cooperative ARQ (DC-ARQ)}

The key idea of this scheme is to delay retransmissions coming from cooperators until the platoon is on a dark area out of coverage of any AP. This scheme operates into three phases, see Fig. 1:

\subsubsection{Association phase}

Vehicles detect AP along the road and register its willingness to download packets. Suitable mechanisms for detecting in-range AP, association and authentication of vehicles reaching a given AP must be provided. Those mechanisms can have a major importance on the overall performance, but are not specific of the use or not of cooperation, and thus we leave them out of the scope of this paper. It can be assumed, for example, that vehicles are equipped with WAVE (Wireless Access in Vehicular Environments) IEEE $802.11 \mathrm{p}$ cards. WAVE architecture provides mechanisms to access WAVE Base Stations (AP) in vehicular networks. In our framework, AP periodically broadcast hello beacons each 0.2 seconds, identifying their zone. Each node that receives the beacon knows it is in coverage and will reply with a registration packet containing its identification.

\subsubsection{Reception phase}

We first describe the basic Packet-by-Packet Cooperative ARQ mechanism and then modified it to consider Delayed Cooperative ARQ. Vehicular nodes receive data from the AP. Each car receives its data but also buffers the packets addressed to other cars in the platoon that consider it as cooperator. The cooperation relationship is established through the exchange of HELLO messages broadcasted periodically by the vehicular nodes. The first function of a HELLO message sent by a node $x$ is to allow other nodes to know about the presence of $x$. Other vehicular node $y$ in the platoon will add $x$ to its list of cooperators (if $x$ is not already a cooperator of $y$ ) when receiving this HELLO message. The second function of a HELLO message sent by a node $x$ is to notify other nodes about the fact that they have to act as cooperators of $x$. For this second function, each HELLO message contains the list of cooperators of the sending node. In our example, the next HELLO message sent by $y$ will contain $x$ in the list of cooperators. In this way, $x$ will be aware of the fact that $y$ considers it as cooperator and will act accordingly (buffering packets addressed to $y$ ).

The list of cooperators contained in the HELLO messages also indicates the order in which cooperators should act when the destination node fails to receive the packet from the AP: this is to avoid collisions; when cooperators detect that the destination node has not received correctly a packet after the ACK timeout, each cooperator will wait a fixed back-off depending on this assigned order, before retransmitting the packet. A retransmission from the AP will be only needed in case that any cooperator 
can not deliver the correct copy of the packet to the destination node. Note that we do not focus on the cooperators selection algorithm, so this is left out of the scope of this document.

For the case of DC-ARQ, cooperators do not retransmit the packets while they are in this phase. Retransmission (i.e. cooperation) of packets will be delayed until the platoon of cars is out of the coverage area in a new phase that we call Cooperative$A R Q$ phase (see next section 3.2.3).

In the considered scenario, data flow is always from the AP to the vehicular nodes, and no retransmissions are used. We avoid retransmissions at the hope that other cars in the platoon (i.e. cooperators) will receive packets incorrectly received by the destination and will help it in the Cooperative-ARQ phase, without the need of wasting the useful time in coverage with the AP in retransmissions. In this way the channel can be used by the AP to transmit as much new data addressed to the cars as possible, thus reducing the downloading time and increasing the effective data rate.

\subsubsection{Cooperative-ARQ phase}

When the cars leave the AP range, they enter into the Cooperative-ARQ phase. After some time (e.g. equivalent to 10 beacons) without receiving beacons from the AP, a node considers that is out of the AP coverage. At this point, every node checks which packets it has failed to receive correctly from the AP and starts to request them to other vehicular nodes (i.e. to its cooperators), in an attempt to recover all packets from the first to the last received from the AP. The process is the following: (i) A node $x$ broadcasts a REQUEST packet for each started block that it has failed to complete from the AP with its packet's received bitmap. (ii) When receiving this REQUEST, each cooperator of $x$ will check if it has any packet from the requested block buffered (it has received the packet correctly from the AP in the previous phase). (iii) If it has some packets, it will send the packets to $x$ (unless other cooperator sends it before).

This process will be repeated while the node receives any packet from its requests, and ends after the tenth time that any cooperator haven't replied or when it enters in range of a new AP, meaning that it comes into reception mode (Reception phase of the protocol operation), and the whole cycle starts again.

Note that the end-to-end block ARQ still is working on top of the cooperative ARQ mechanisms. The scheme trade-offs among delaying the recovery of packets from cooperators until vehicles reach dark areas in which there are no AP contacts and recovering the packets from the higher layer ARQ protocol.

\section{Performance of Delayed Cooperative ARQ}

The simulations were performed with the standard version of the ns-2.31 simulator [2]. Each AP generates packets to vehicles in its coverage area in round robin basis. Vehicles request files of $10 \mathrm{MB}$ and packets have a size of $1 \mathrm{~KB}$.

Vehicles travel $30 \mathrm{Km}$ on a two-way highway with two lanes per direction. The road network infrastructure consists of 5 Access Points placed every $6 \mathrm{Km}$. Vehicles move with constant speed, randomly chosen from a uniform distribution between 70$90 \mathrm{~km} / \mathrm{h}$ on the right lane and between $90-120 \mathrm{~km} / \mathrm{h}$ on the left lane. Vehicle density 
is modeled with exponential distribution of parameter $\lambda_{1}$ vehicles/s in the right lane and $\lambda_{2}$ vehicles/s in the left lane. These rates consider the maximum number of vehicles following vial rules (e.g. security distances of 80 meters traveling at 90 $\mathrm{Km} / \mathrm{h}, 100$ meters traveling at $100 \mathrm{Km} / \mathrm{h}$, etc). Using these consideration, $\lambda_{1}=0.25$ vehicles/s in the right lane and $\lambda_{2}=0.2$ vehicles/s in the left lane would fill the highway with the maximum number of vehicles traveling at $100 \mathrm{Km} / \mathrm{h}$ and at 120 $\mathrm{Km} / \mathrm{h}$ at each lane. Higher densities may be achieved lowering vehicle speeds (then the security distance between vehicles is lower). In the graphs we normalize $\lambda_{i}(i=1,2)$ and for clarity we define parameter $\alpha$ as vehicle density, being $\alpha=1$ the higher density corresponding to vehicles with $120 \mathrm{Km} / \mathrm{h}$ as maximum speed at line 1 and $90 \mathrm{Km} / \mathrm{h}$ as maximum speed at line 2 . Lower values of $\alpha$ indicate a decrease in vehicle density.

For the physical layer we consider IEEE802.11a at a rate of $3 \mathrm{Mb} / \mathrm{s}$. Nodes use omni-directional antennas, see Table 1.

Table 1. Configuration parameters

\begin{tabular}{|l|l|}
\hline Antenna & Omnidirectional \\
\hline Frequency & $5.9 \mathrm{GHz}$ \\
\hline RxTh & $-95 \mathrm{dBm}$ \\
\hline CSTh & $-96 \mathrm{dBm}$ \\
\hline Antenna Gain & $2.512 \mathrm{~dB}$ \\
\hline TxPower & $9.95 \mathrm{dBm}$ \\
\hline
\end{tabular}

We have used Nakagami as propagation model. This model is used to predict signal attenuation in fading environments and has already been used in vehicular scenarios, [14]. The Nakagami probability density function, [15], defines a distribution of the power $\mathrm{x}$ of the received signal:

$$
f(x ; m, \Omega)=\frac{m^{m}}{\Gamma(m) \Omega^{m}} x^{m-1} e^{-\frac{m}{\Omega} x}
$$

Where, $\Gamma$ denotes the Gamma Function, $\Omega$ denotes the average received power, $\mathrm{m}$ is the Nakagami parameter and both $\Omega$ and $\mathrm{m}$ depends on the distance between transmitter and receiver. High values of the $m$-parameter (with $m>1$ ) introduces a variability on the average power reception similar to two ray-model while a value of $\mathrm{m}=1$ introduces Raleigh distribution variability. Lower values of the parameter $\mathrm{m}$ worsen channel performance. That allows to define a reception similar to two-ray mode in short distances with reception powers that depends on the $\mathrm{d}^{-2}$ and reception similar to fading models in larger distances with reception powers that depends on $\mathrm{d}^{-\gamma}$ $(\gamma>2)$.

Figure 2 shows delivery ratio for of two path loss scenarios and two antenna gains. The scenarios considered are specified in Table 2. Scenario A considers a two-ray model in short distances $(\mathrm{d}<200$ meters $)$. At distances higher than 200 meters, the reception power decreases at a power of $\gamma>3.8$. The fading channel is the same at all distances: a high loss fading channel of $m=0.5$. Scenario $B$ is very similar to scenario A, but with different fading channel depending on the distance. Distances lower than 80 meters have a Raleigh fading channel, from 80 to 200 meters the channel is worst $(\mathrm{m}=0.75)$ and finally at higher distances $(\mathrm{d}>200$ meters $) \mathrm{m}=0.5$ (an even worst fading channel). As a summary Scenario A is more pessimistic than Scenario B. 
Table 2. Fading Scenarios

\begin{tabular}{|l|l|c|c|c|}
\hline & & $\mathrm{d}<80$ & $80<\mathrm{d}<200$ & $200<\mathrm{d}$ \\
Scenario A & $\mathbf{m}$ & 0.5 & 0.5 & 0.5 \\
& & $\mathrm{~d}<200$ & $200<\mathrm{d}<500$ & $500<\mathrm{d}$ \\
& $\boldsymbol{\gamma}$ & 1.9 & 3.8 & 3.8 \\
\hline & & $\mathrm{d}<80$ & $80<\mathrm{d}<200$ & $200<\mathrm{d}$ \\
Scenario B & $\mathbf{m}$ & 1.0 & 0.75 & 0.5 \\
& & $\mathrm{~d}<200$ & $200<\mathrm{d}<500$ & $500<\mathrm{d}$ \\
& $\boldsymbol{\gamma}$ & 1.9 & 3.8 & 3.8 \\
\hline
\end{tabular}

Remember that the $\alpha$ depicted on the $\mathrm{x}$-axis means the normalized car density with respect to the maximum possible. As expected, results show a worst delivery ratio on scenario A and a straight line in all the scenarios indicating independence with respect the vehicle density. From Fig. 2 it can also be observed the impact of the antenna gain in the delivery ratio.

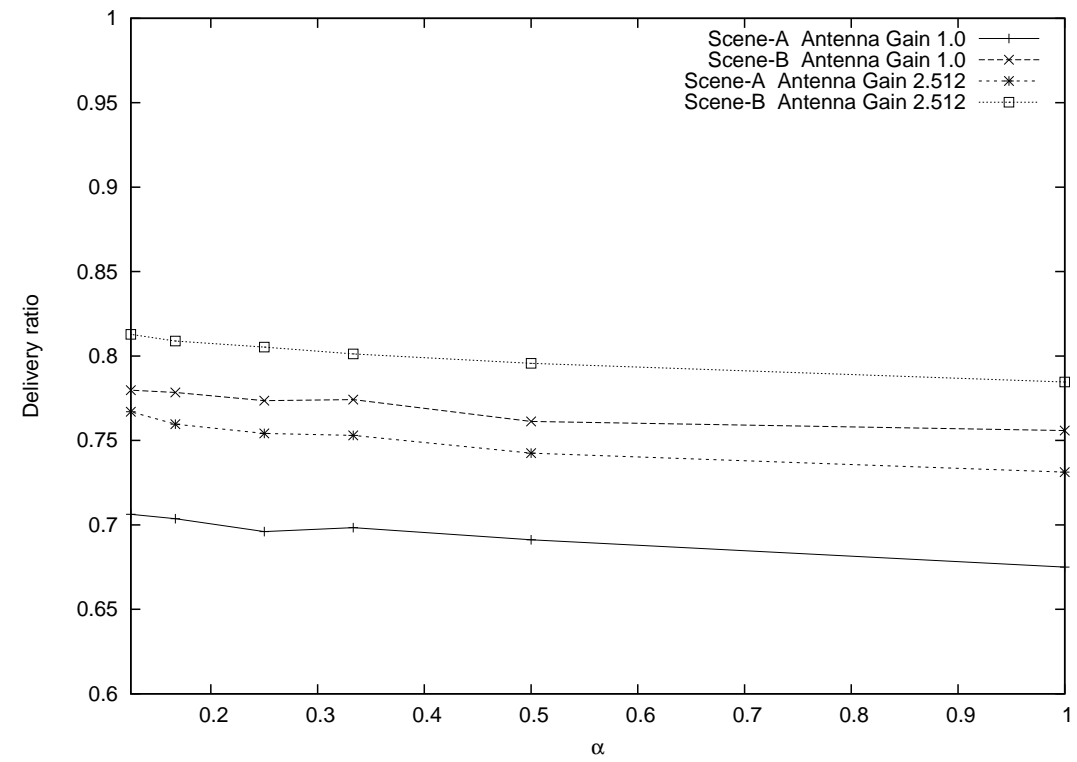

Figure 2. Delivery ratio as a function of car density

Figure 3 shows the packet delivery ratio as a function of vehicle density for the baseline case in which no cooperation is used and for the case in which the proposed cooperation scheme (DC-ARQ) is utilized. The two curves correspond to Scenario B with an antenna gain of $1 \mathrm{dBm}$ (these parameters are the defaults in ns-2 for the Nakagami channel). The first curve (i.e. no cooperation) shows almost a straight line indicating that packet losses are independent of vehicle density, as seen on previous Figure 2. That means that as long as we are not using other cars to improve the reception of the receivers, they do not affect to the overall delivery ratio. Note the 
difference with the case of using DC-ARQ: in this case, as long as the number of cars on the road (i.e. vehicle density) increases, receiving nodes can benefit from cooperation. Note that at maximum node density DC-ARQ allows for an improvement of the delivery ratio from 0.76 , in which no cooperation is used, to 0.96 . Also interesting, is the improvement that DC-ARQ allows even for lower densities.

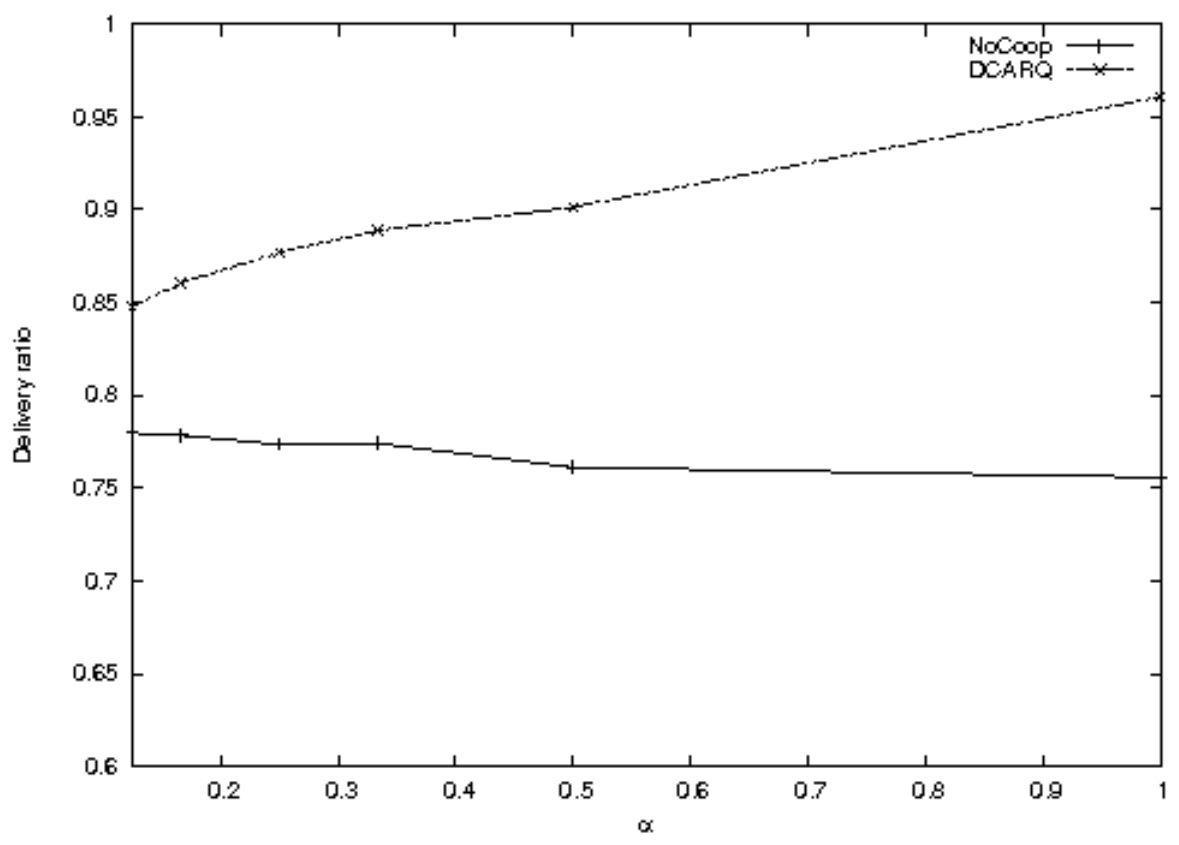

Figure 3 Delivery ratio as a function of car density (Scenario B)

Figure 4 depicts a slightly better scenario in which the parameter $m$ is set to 1 for all the distances (i.e. cars are always facing a Rayleigh channel). We can observe the same behavior than that in Figure 3. The only difference is that as long as the channel conditions are better, both curves (i.e. no cooperation and DC-ARQ) perform better). It is interesting to see, however, how the delivery ratio of DC-ARQ approaches 1 in this case for a full node density. More scenarios have been simulated, and in all cases the same behavior has been obtained. 


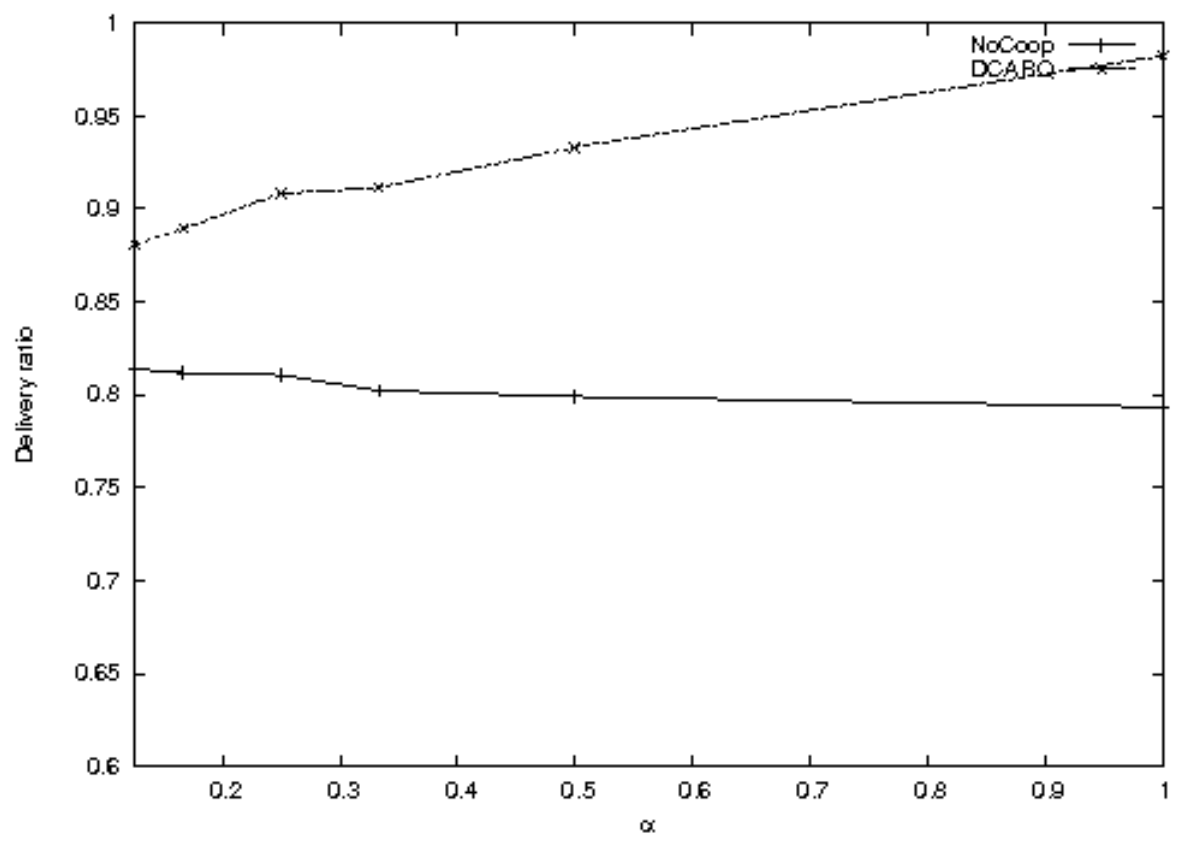

Figure 4 Delivery ratio as a function of car density $(m=1)$

From Figures 3 and 4 we may extract some interesting conclusions: (i) DC-ARQ allows the recovery of lost packets from neighboring vehicles. This recovery may be performed in dark areas in which there is not Internet access. In this way, use of bandwidth during contact times is optimized. (ii) DC-ARQ recovers packets but is not an end-to-end ARQ mechanism. That means that is not able to guarantee the whole reception of a file. (iii) DC-ARQ is able to recover with high probability almost all packets when vehicle density is higher. The idea is that in high vehicle density scenarios there always are vehicles in the AP communication range. These vehicles will receive packets destined to other vehicles with high probability and afterwards cooperate with them to deliver them. This can be seen in Figures 3 and 4 where in high scenarios the recovery of packets is quite high. However, in low vehicle density scenarios, there are few or none vehicles in the AP communication range that may receive packets destined to other vehicles. In this case, waiting to the end of the communications range to recover packets wastes the possibility to recover packets using an end-to-end ARQ during AP contact times. In any case, an end-to-end ARQ mechanism is needed to recover those lost packets, even in high vehicle density scenarios.

From these conclusions, we think in three possible solutions to analyze in further studies: (a) an adaptive scheme in which AP switch from end-to-end ARQ to DCARQ when vehicle densities are high on its coverage. The end-to-end ARQ would be enabled when vehicles enter next AP coverage. (b) using different channels in which reception of information and cooperation is established. (c) Network coding would allow synchronization in the reception of packets and therefore would add robustness. 


\section{Conclusions}

This paper has evaluated Delayed Cooperative ARQ (DC-ARQ) mechanism in a Vehicular Ad Hoc Network (VANET). The paper analyzes a Delay/Disruptive Tolerant Network scenario in which vehicles download information from access points distributed along roads. Optimizing the use of bandwidth during contact times is a key factor in this kind of networks. DC-ARQ waits until vehicles leave AP coverage to cooperate in dark areas not wasting AP bandwidth resources in recovering packets. Simulation shows that DC-ARQ may recover high amount of packets in high vehicle density scenarios. However, in low vehicle density scenarios cooperation is not able to recover too many packets. This is due to the lack of cooperators. In these cases, contact times should be used to download as much as possible information together with an end to end recovery mechanism.

\section{References}

1. J. Ott, D. Kutscher, "Drive-thru Internet: IEEE 802.11 b for 'Automobile' users", IEEE INFOCOM 2004.

2. Network Simulator ns-2. http://www.isi.edu/nsnam/ns/.

3. J. Burguess, B. Gallagher, D. Jensen and B. N. Levine, "MaxProp: Routing for Vehiclebased Disruption Tolerant Networks", $25^{\text {th }}$ Conference on Computer Communications (IEEE INFOCOM), Barcelona, Spain, April 2006.

4. A. Balasubramanian, B. N. Levine and A. Venkataramani, "DTN Routing as a Resource Allocation Problem", ACM SIGCOM'07, Kyoto, Japan, August 2007.

5. J. Zhao and G. Cao, "VADD: Vehicle-Assisted Data Delivery in Vehicular Ad Hoc Networks", $25^{\text {th }}$ Conference on Computer Communications (IEEE INFOCOM), Barcelona, Spain, April 2006.

6. Vahdat and D. Becker, "Epidemic Routing for Partially Connected Ad Hoc Networks", Technical Report CS-200006, Duke University, April 2000.

7. Bin Zhao, Matthew C. Valenti, "Practical Relay Networks: A Generalization of HybridARQ”, IEEE Journal on Selected Areas in Communications, vol. 23, no. 1, Jan. 2005.

8. Miu, H. Balakrishnan, C. E. Koksal, "Improving Loss Resilience with Multi-Radio Diversity in Wireless Networks", ACM Mobicom 2005, September 2005.

9. M. Dianati, X. Ling, K. Naik, X. Shen, “A Node Cooperative ARQ Scheme for Wireless Adhoc Networks", IEEE Wireless Communications and Networking Conference, 2005.

10. J. Morillo, J. Garcia-Vidal, "A Low Coordination Overhead C-ARQ Protocol with Frame Combining”, $18^{\text {th }}$ Annual IEEE International Symposium on Personal, Indoor and Mobile Radio Communications (IEEE PIMRC 2007), Athens, Greece, September 2007.

11.J. Morillo-Pozo, O. Trullols, J. M. Barceló, J. García-Vidal, "A Cooperative ARQ for Delay-Tolerant Vehicular Networks", to be published in DTMN 2008.

12. T. Small and Z. J. Haas, "The Shared Wireless Infostation Model - A new Ad Hoc Networking Paradigm (or Where there is a Whale, there is a Way)", MobiHoc'03, Annapolis, Maryland, USA, June 2003.

13. K. Fall, "A Delay-Tolerant Network Architecture for Challenge Internets", IRB-TR-032003, Intel Research Berkeley, Feb 2003

14. M. Torrent-Moreno, "Inter-Vehicle Communications: Achieving Safety in a Distributed Wireless Environment”, Dissertation, Universitätsverlag Karlsruhe, 2007.

15. W. Zhang and N. Moayeri, "Classification of Statistical Channel Models for Local Multipoint Distribution Service using Antenna height and directivity", IEEE 802.16 working group contribution IEEE802.16.1pc00/07, Jan 2000 